\title{
LA EXISTENCIA SOCIAL: entre subjetividad y condiciones objetivas - Incidencia en la discusión teórica sobre el desarrollo
}

\author{
Aura González Serna \\ Universidad Pontíficia Bolivariana (UPB)
}

LA EXISTENCIA SOCIAL: entre subjetividad y condiciones objetivas - Incidencia en la discusión teórica sobre el desarrollo Resumen: Este artículo busca instigar reflexiones sobre el debate teórico en torno al Desarrollo. La premisa para afincar el análisis es considerar la incidencia de los modos de aprehender la existencia social, toda vez que ella está demarcada entre subjetividades y condiciones objetivas. Concepciones distintas al interpretarla y representarla comparecen en el imaginario individual y colectivo. La fragmentación entre subjetividad y objetividad constituye impedimento para comprender el carácter genérico del Ser que construye y transforma permanentemente la existencia social.

Palabras-clave: Desarrollo, existencia social, relación naturaleza-sociedad

THE SOCIAL EXISTENCE: Between subjectivity and objective conditions. Incidence In theoretical discussion about development

Abstract: This article aims to investigate reflections about the theoretical debate about development. The premise to establish the analysis is to consider the incidence in the apprehend ways the social existence every time it is demarked among subjectivities and objective conditions. Different conceptions to interpret and represent it show in the individual and collective imaginary. The fragmentation between subjectivity and objectivity constitute impediment to comprehend the generic character of the being that build and permanently transform the social existence.

Keywords: Development, social existence, nature-society relation. 
1 RELACIÓN NATURALEZA - SOCIEDAD: dialogo entre materialidad y subjetividad

Conceptos tales como: la relación naturalezasociedad, el espacio, el paisaje, el territorio, el desarrollo, el capital humano, el capital ambiental, la sustentabilidad, el medio ambiente, entre muchos otros, son instrumentalizados a partir de innumerables demandas, buscando operar a través de adjetivos o adverbios que califican racionalidades y decisiones.

Influencias teóricas incidirán en estas trayectorias. Por ejemplo, en Hegel el paisaje será entendido como una subjetividad objetivándose a través del trabajo, o para decirlo de otra manera, una interioridad exteriorizándose en lo que se denomina paisaje, al buscar reconocerse en él. $Y$ de este modo, otorgándosele cualidades estéticas que no podrían comprenderse sin considerar el diálogo entre materialidad y subjetividad.

Esta objetivación del espíritu en la naturaleza se da, para Marx, a través del trabajo que transforma la naturaleza. Es por esto que el paisaje, donde se ha cristalizado el trabajo humano, muestra los modos de producción que lo han caracterizado a lo largo del tiempo.

Este ejemplo, la idea Hegeliana sobre la naturaleza como síntesis de una interioridad exteriorizándose, estará asociada a los contenidos simbólicos de los objetos que constituyen el paisaje y tendrán vasta influencia en la Geografía alemana y cultural.

En este mismo sentido, debatir sobre concepciones del Desarrollo estará relacionado con la coyuntura histórica que lo concibe, e implica identificar vínculos que estructuran continuidades y rupturas en la dimensión socio-territorial, referida ésta al espacio geográfico, en cuanto contexto en el cual se revelan modos de producción y relaciones sociales en realidades especificas. Condición que ha sido bastante desestimada en la concepción neoliberal.

En el capitalismo, el hecho que los procesos productivos se consiguieran segmentar, funcional y espacialmente, ganando rentabilidad y eficiencia, llevó a muchos autores a plantear la pérdida de la centralidad del trabajo en la organización de la sociabilidad. Se acuñaron conceptos como sociedad de la información, los no-lugares, deslocalización, sociedad pos-industrial. En el contexto de la globalización, resurge la relevancia del ser humano en territorios específicos, asociando recursos, generación, transmisión de conocimientos, competencias e innovación.

En el modo actual de realización del capital, su reproducción reclama anclarse, influir directamente en modos de vida cotidiana, favorecerse de contenidos en la relación entre el conocimiento y el territorio. Con esto se opera un movimiento que supera las ilusiones de afianzarse sobre imaginarios de lo volátil y asequible, en condición de itinerante. El capitalismo estimula multiplicidad de significados y adjetivos, en cuanto a definiciones e ideologías que buscan construir consensos mediadores para legitimar modos de operar. Asistimos a una racionalidad que requiere territorializar la globalización. Políticos y economistas insinúan que las relaciones humanas son naturales, inmutables, típicas de sociedad en abstracto, estimuladas por la competencia entre individualidades. El concepto de desarrollo sostenible busca nuevos argumentos, ante las manifestaciones de la naturaleza, determinada por un conjunto de condiciones históricamente constituidas y agravadas en la densidad y tecnicidad por las realizaciones humanas recurrentes, aunado a ciclos del metabolismo que rigen los elementos de la naturaleza, en una fase en la que diversas catástrofes socio-ambientales, visibilizan problemáticas globales.

Compartimos el parecer de Medeiros \& Sá Barreto (2012) cuando destacan que autores como Foster (2002) y Burkett (1999) establecieron con éxito el vínculo entre la dinámica propia de la formación socio-económica vigente y los innumerables fenómenos de degradación ambiental. Esa manifestación de la degradación ambiental, profundizada por la crisis del capitalismo global, ha resultado emblemática para ideologías y prácticas, a saber, la del ecologismo: estudio científico de la relación entre la vida social y el ambiente natural. Sus propósitos, en este contexto demarcados entre particularidades históricas, requieren superar preceptos éticos abstractos, en el sentido de ser postulaciones, que necesitan establecer relaciones con los presupuestos y problematizaciones objetivas de las prácticas sociales cotidianas, en los espacios que le dan forma. Puesto que al reproducir una ideología favorable a preceptos que se metabolizan en discursos, terminan favoreciendo la manutención del sistema capitalista.

\section{LOS VALORES Y LAS PRÁCTICAS SOCIALES}

Más allá de ser preceptos éticos abstractos, es prioridad que se distinga la imposibilidad de superar problemas ambientales al interior de la formación social regida por el capital, en el modo como se realiza el consumo y la generación de lucro. Esto remite a la concepción de valores en cada coyuntura histórica.

Los valores son entidades subjetivas y condiciones objetivas de la práctica social, cuyas formas de realización requieren ser deconstruidas, en la búsqueda por evidenciar la incompatibilidad entre lo que se divulga en el plano ético-moral y las realizaciones humanas contemporáneas. Es decir, postulaciones enmarcadas en racionalidades que sistematizan bagajes teóricos requieren ser investigadas a la luz de las prácticas sociales que le dan forma en coyunturas especificas. Siendo que 
en la sociedad regida por el modo de explotación capitalista, todos los valores, inclusive los que postulan la denominada ética ambiental, tienen su realización subordinada a una ética que requiere naturalizarse e ideologizarse como ética objetiva (o autónoma): la ética del capital.

Las abstracciones racionalizadas de la ética ambiental adquieren, para descascarar sus artificios, el peso de expresión práctica, de aprehensión dialéctica y de análisis de totalidad, entre lo concreto vivido y lo concreto pensado (apoyándonos no solo en la crítica social de Marx, sino también en la amplia expresión de la subjetividad en lo contemporáneo, recurriendo a Lukács).

\section{CONCEPCIONES AFINES AL CAPITALISMO}

Revisando la construcción teórica que desde perspectivas diversas ha venido observando la degradación ambiental y el desarrollo del capitalismo, se encuentra en la economía una vertiente ortodoxa, que apoyándose en el legado de los clásicos, atribuye a la ausencia de incentivos de mercado la causa de los principales problemas ambientales.

En esta perspectiva, el modelo de explotación y realización de lucros del capitalismo no se cuestiona, debe quedar intacto, pero ampliando la extensión de la lógica del mercado hacia realidades socioespaciales en las cuales este no viene operando de modo eficiente. Este metabolismo generaría un corrector, donde, se cree, que los individuos serian estimulados a reducir sus prácticas dilapidadoras hacia sentidos/valores sustentables.

Existe otra corriente que prefiere interpretar la problemática ambiental en términos alternativos, se defiende la producción y el consumo conscientes, con frenos: parafraseando sus principios serian buenas prácticas, a través del enfoque de prácticas individuales se asentaría una transformación ética generalizada. Aquí se podría cuestionar la extensión de este compromiso individual con el medio ambiente, de cierto cuño teológico, moral, idealista, de un modo que parece tranquilizar los sentidos, sin comprender que la tentativa afianza la lógica individualista que metaboliza el modo de realización del capital, compréndase en este sentido la moda o énfasis por las ferias ecológicas, la agricultura orgánica que pauta publicidad y consumo más costoso, la homogenización de un discurso que se impone en la expresión tácita, mas no en los factos. Dinámica que metamorfosea en alienación y mercancía el proceso, pues nadie termina preguntándose de dónde vienen las legumbres, las frutas, el modo de trabajo que compromete cada producción.

Recuperaremos a continuación el contexto que ha favorecido esta problematización.

Parafraseando a Smith, el autor expresa la posibilidad de cuestionar el crecimiento económico como fin en sí mismo y el impulso a la ampliación irrestricta de la producción cambiando un determinado conjunto de valores. Sería necesario, según él, orientar estos valores hacia las reales necesidades de los seres humanos, superando el sentido anti ecológico y el uso de avances tecnológicos de forma dilapidadora. Habría que poner freno a las representaciones del dominio de la humanidad sobre la naturaleza, ya que esta concepción conlleva un carácter dual: mientras ofrece comodidades al responder a necesidades, al mismo tiempo, de modo imprevisible e irreversible transforma el carácter de la naturaleza.

Es evidente la interacción e indisociabilidad establecida entre naturaleza y sociedad en las formulaciones económicas del siglo XIX e inicio del XX. Paul Claval (2005) rescata que el conocimiento de la economía no se desarrolló hasta final del siglo XVIII, gracias a la observación del papel del espacio en la vida económica. Esto también fue demostrado por Pierre Dockes (1969). Estaría evidenciando marcos de la economía y geografía espacial, a partir de la reflexión sobre los problemas de la riqueza y la producción. Nace la observación del paisaje y la necesidad de representar la realidad geográfica. Al final del siglo XVII, economistas como William Petty, en Inglaterra, y Vauban, en Francia, ya identificaban que las actividades productivas estaban generalmente concentradas a lo largo de los litorales, de los ríos navegables y de los canales. En el siglo XVIII la atención se concentró cada vez más sobre la producción y el intercambio de riquezas. Richard Cantillon describió la creación de riquezas en las zonas rurales y su consumo en las ciudades: de este modo ofreció la primera reflexión sobre la naturaleza de la ciudad y la jerarquía urbana. Quesnay, economista francés, fue por su parte, el primero en concebir la vida económica como un circuito. Después de 1770 la situación cambió. Los economistas desarrollaron un interés creciente por los mecanismos económicos. Turgot describió el funcionamiento de los mercados y la ley de oferta y demanda.

En la Riqueza de las Naciones, Smith (1776) manifestaba el interés por la observación geográfica, demostrando que la especialización del trabajo era limitada por la extensión del mercado. Sin embargo, Smith consideró que la riqueza de las naciones resultaba de la voluntad de los individuos, de las iniciativas de los emprendedores y del libre funcionamiento de los mercados, de ahí viene la exclamación: dejar hacer, dejar pasar!. Se identifica que a partir de estos postulados cesa el estimulo a identificar particularidades de las distribuciones geográficas.

El economista evidenció la combinación de los factores productivos en la empresa y la responsabilidad de los gobiernos en el campo de funcionamiento de las firmas y de los mercados. El problema mayor estaba en la repartición de las rentas entre capitalistas y trabajadores. El ramo principal de la ciencia económica ignoró el papel 
del espacio por más de un siglo, hasta la década de 1930.

En Alemania, al decir de Paul Claval (2005), Ernst Friedrich introdujo en 1904 la noción economía explotadora y destructiva. Hasta el fin del Siglo XIX, el libre comercio fue efectivamente la causa del desarrollo económico de Europa Central y Occidental y de Estados Unidos. En esta perspectiva, la posición de David Ricardo (1817) estaba correcta: en una economía sin progreso técnico, es decir, sin economías de escala y sin economías externas, el comercio internacional favorece el nivelamiento de los precios de los factores de producción, la mejor política era el libre comercio.

Sin embargo, será a partir de la década de 1970 que estos postulados entran a hacer parte de las agendas internacionales, a modo de reconocimiento, urgencia y definiciones que tensionan la definición de políticas públicas. En este sentido, el llamado tercer sector asumió representativamente el propósito de modificar hábitos de consumo y de incidir en nichos de la producción. Identificando intencionalidades de una coyuntura, inseparables a las acciones que configuran tensiones, Al Gore, ex vicepresidente norteamericano, ante la crisis ambiental manifestaba, por ejemplo:

[...] es fácil sentirse masacrado e impotente, no confiar en que los esfuerzos individuales puedan tener real impacto. Necesitamos resistir a esa reacción, esta crisis no va a tener fin si nosotros, como individuos, no asumimos la responsabilidad por el problema. Procurando informarnos e informar a los otros, volviéndonos más activos políticamente y exigiendo cambios, cada uno de nosotros puede hacer la diferencia. (GORE, 2006, p. 317).

La Comisión Mundial sobre el Medio Ambiente y Desarrollo (CMMAD, 1991) considera que el desarrollo sustentable debe ser un proceso capaz de armonizar crecimiento económico, inversiones, avance tecnológico, con la explotación de los recursos y del medio ambiente en general. Para Schumacher (1996) es necesario transformar los valores asociados al consumo como condición sine qua non para una sociedad sustentable. Recurre al budismo y al cristianismo para explicar su concepto de consumo consciente, basado en simplemente el freno al deseo por más. Tal revisión de valores (ética) y comportamientos (moral) generarían modelos sustentables, que invertirían las tendencias actuales de la degradación ambiental.

De ese modo, se analiza el camino propuesto por estas reflexiones y autores. No se niega que esas exposiciones cumplen un papel importante al evidenciar modelos actuales de producción, consumo, comercialización, distribución, contaminación y atentado contra recursos naturales no renovables. El llamado es a vincular la existencia social entre subjetividad y condiciones objetivas. La ética y la moral no se forman de modo libre e irrestricto en la conciencia de los individuos. No será a partir de valores y meras intenciones que se configura la vida cotidiana y su red de relaciones hacia modos diversos de sociabilidad.

Autores diversos han contribuido al debate sobre la idea de que entre la sociedad y el producto materializado en acciones, existe una trama de intencionalidades, por lo que este continuum entre el producto materializado en acciones es inseparable de las intencionalidades que lo rigen. De ese modo, la ética no es exclusivamente subjetividad. Siendo ética y moral, caras de una misma moneda, en el sentido de las determinaciones que los modos de reproducción social le impregnan.

4 LA EXISTENCIA SOCIAL: entre lo inmanente y trascendente

No hay duda que para romper esta dualidad y fragmentación al referirse a la ética, hay que recurrir a la ontología de los valores, identificando que los valores son momentos que no se pueden eliminar de las prácticas humanas. Lukács realiza un ejercicio detenido examinando las formas de prácticas humanas originarias, a través de una revisión a la acción humana a partir del trabajo. Observa que éste se constituye, históricamente, en esencia de objetivación con una previa definición (teleología), buscando materializar la idea. Independiente de cualquier forma histórica concreta, es una práctica que busca objetivar un valor-deuso o simplemente un valor. Lukács reconoce que una vez puesta la finalidad que conduce al trabajo es necesario que el proceso de trabajo sea compatible con su objetivación. Eso significa que el nexo entre el valor y el deber-ser ya está presente como momento indispensable del trabajo, incluso en sus formas más primitivas. Siendo el trabajo una actividad programática y de realización, su producto es objetivación, simultáneamente, de las manos y del cerebro, de las aptitudes corporales e intelectuales del hombre, Markus (1974). El trabajo, siendo un acto de accionar consciente, pone y supone el conocimiento de finalidades y medios. Todo trabajo implica un cierto saber sobre los hombres en sus relaciones sociales y personales, como condición de inducir el sujeto a efectuar los propósitos deseados. Este saber asume las formas de costumbres, hábitos, tradiciones, evidenciándose en procedimientos racionalizados. De este modo, el trabajo es inseparable del conocimiento, de las ideas y concepciones de mundo, esto significa, de formas de pensar la vida real. El ser que trabaja construye para sí, a través de su actividad, modos de actuar $y$ de pensar, es decir, una manera específicamente humana de relacionarse con las circunstancias objetivamente existentes, apropiándose de ellas, 
trazando la consecución de fines propuestos por el sujeto en la creación de objetos capaces de desempeñar funciones sociales, haciendo nacer valores de uso.

El hecho de contener una ética (valor) y una moral (deber-ser), distingue la práctica humana del trabajo (y la práctica general) de las actividades análogas de los animales. En la naturaleza, dice Lukács, hay emergencias y satisfacciones, pero no valores. Lukács (1979, p. 86; 2004, p. 143).

[...] toda praxis, incluso la más inmediata y la más cotidiana, contiene en sí esa referencia al acto de juzgar, a la conciencia, etc., visto que es siempre un acto teleológico, en el cual la posición de la finalidad precede, objetiva $y$ cronológicamente, la realización.

Surge una categoría fundamental para Lukács en la praxis humana: la alternativa. El trabajo además de ser realización de una finalidad preconcebida, es elección entre alternativas concretas. En todo acto humano no solo existe la finalidad (valor) sino un curso de acción (deber-ser) y todos los otros medios necesarios para realizarla objetivamente en un mundo en sí insensible en relación a los designios humanos, son elegidos, y otros negados. Reconocer ese carácter de elección entre alternativas nos permite revelar el fundamento objetivo de los valores y a partir de ellos, de los deberes-ser y juicios de valor.

De este modo, se puede analizar que la propia elección depende del hecho de que el mundo contenga en su configuración objetiva posibilidades todavía no explicitadas y que jamás se explicitarían a no ser por la realización exitosa de la práctica humana. Por lo tanto, es imprescindible que los sujetos, en su práctica, reconozcan y elijan los cursos de acción que se ajustan a la realización de la finalidad puesta al inicio idealmente. Es a través de este ejercicio del razonamiento que Lukács demuestra el carácter objetivo de los valores, en la demostración del carácter objetivo del deber-ser y los juicios de valor.

El trabajo, Lessa (2002), es el punto de partida de la humanización del hombre, del refinamiento de sus facultades, proceso del cual no se debe olvidar el dominio sobre sí mismo. Por mucho tiempo el trabajo fue el único ámbito de ese desarrollo. En este sentido aquí el trabajo es tomado como modelo ontológico de las diferentes modalidades de práctica social. Por el trabajo se transforman causalidades insensibles a las finalidades humanas en causalidades "puestas" por su práctica. Y los seres humanos en el contínuum de este proceso crean en el mundo nuevas formas materiales. Eso quiere decir que los seres humanos modifican el campo de los valores y, a través de esto, de los deberes-ser y de los juicios de valor. A cada etapa del desarrollo social, corresponde no sólo un conjunto de valores, una ética, sino una moral y formas de subjetividad (pueden conocerse como ideología).

Así, para conocer la ética y la moral de una determinada época o coyuntura histórica, se deben evidenciar los condicionantes de la práctica humana determinados por el grado de desarrollo social. $Y$ aquí se explica la crítica a la ética idealista, con base en la teoría del valor de Marx y en el estudio de la ontología y la subjetividad desarrollado por Lukács. Con el vinculo insuperable entre ética, práctica social y estructuras causales que constituyen el mundo de los hombres.

\section{CONCLUSIONES}

Retomando la discusión teórica sobre el Desarrollo, en su relación dialéctica entre naturaleza \& sociedad, el desafío es aprehender/analizar los modos diversos de territorialidad que se ejercen, las redes que denotan movilidad y tácticas a veces inverosímiles y desestimadas, del hombre a través de la reproducción cultural, en el ámbito cotidiano. La naturaleza aquí evocada no en calidad de primera naturaleza y sí en su condición de segunda naturaleza, referida a la relación de los sujetos que explotan los recursos del medio, generando cambios que resultan en infraestructuras, comunicaciones, edificaciones, equipamientos e instituciones, a través de un contínuum de relaciones sociales que se manifiestan en discursos, prácticas, representaciones, intencionalidades y producción de sentidos. La naturaleza intervenida como mercancía, desde múltiples sutilezas que imponen innovación, estética, envejecimiento previsto, arreglado y programado dentro de la lógica del objeto obsoleto, en la dinámica contemporánea del capitalismo. La naturaleza se transforma en función de la novedad, para excitar a los compradores a determinados usos con la acotación en el mercado, siendo la publicidad un mecanismo clave en ese proceso Lefebvre (1991).

Lo cierto es que existe un vínculo de dependencia profunda e histórica del hombre por la naturaleza. Resultando en equívoco tanto restringir esa comprensión a una naturaleza esencialmente técnica e instrumental, como el estudio de la naturaleza per se, sin situarla en el movimiento sociogeo-histórico y económico del modo de producción capitalista, en su carácter multidimensional y multiescalar. El carácter de temporalidad establece periodos significativos que permite comparaciones, identificando contradicciones entre los intereses del capital y las prácticas que expresan el ordenamiento de un territorio en contravía de las determinaciones en la relación sociedad-naturaleza, sobre las condiciones referidas al capital, el trabajo, la tierra, la cultura, técnicas y tecnologías.

Comprender que en todo acto humano no solo existe una finalidad (valor), sino un curso de 
acción (deber-ser) y todos los medios necesarios para realizarla objetivamente se realizan entre elecciones y negaciones (la alternativa), es comprender que el mundo contiene en su configuración objetiva, posibilidades aun no explicitadas, múltiples redes que se establecen (hombre-naturaleza, hombrehombre, sociedad-sociedad) y objetivaciones que solo se podrán explicitar a través de la realización de las más diversas formas de uso y ocupación del suelo. Se trata de reconstruir la historia de la producción social del espacio, evadiendo la distracción a sentencias que caracterizan procesos, relaciones sociales y dinámicas como siendo locales, regionales, metropolitanas. La conclusión es que el fundamento objetivo de los valores es la labilidad propia de la existencia tanto natural como social, es decir, que la elección entre alternativas también envuelve el curso de acción en el trabajo, el proceso de trabajo. Es imprescindible que, en su práctica, los sujetos reconozcan/elijan acciones que se ajusten a la realización de la finalidad que al inicio es puesta idealmente. Es un continuum de carácter objetivosubjetivo, del deber-ser y de los juicios de valor.

\section{REFERÊNCIAS}

BURKETT, P. Marx and nature: a red and green perspective. Nueva York, St Martins's Press, 1999.

CLAVAL, Paul. Geografia econômica e economia. GeoTextos, Salvador, v. 1, n. 1, p. 11-27, 2005.

FOSTER, J. B. Ecology against capitalism. Nueva York: Monthly Review Press, 2002.

GORE, A. Uma Verdade Inconveniente. São Paulo: Editora Manole, 2006.

LEFEBVRE, The Production of Space. N. DonaldsonSmith trans. Oxford: Brasil Blackwell, 1991.

LUKÁCS, G. Ontologia do Ser Social. São Paulo: LECH, 1979.

LESSA, S. O Mundo dos Homens: trabalho e ser social. São Paulo: Boitempo, 2002.

MARKUS, G. Teoria do Conhecimento no Jovem Marx. Rio de Janeiro: Paz e Terra, 1974.

MEDEIROS, Joao Leonardo; SA, Barreto Eduardo. Lukács y Marx contra el ecologismo acrítico: por una ética ambiental materialista. Revista Herramienta, Buenos Aires, n. 50, 2012. Disponible en: <http:// www.herramienta.com.ar/herramienta-web-6/ lukacs-y-marx-contra-el-ecologismo-acritico-poruna-etica-ambiental-materialista>. Acceso en: 29 oct. 2012.
SMITH, G. A. The Purpose of Wealth: a historical perspective. In: DALY, H.; TOWNSEND, K. Valuing the Earth: economics, ecology, ethics. Cambridge, MA: MIT press, 1996.

\section{Aura González Serna}

Assistente Social

Doutora em Serviço Social

Professora da Universidade Pontifícia Bolivariana - UPB

E-mail: aura.gonzalez@upb.edu.com

Universidade Pontifícia Bolivariana - UPB

Medellín, Antioquia, Colombia 